\title{
SURFING IN SOUND: SONIFICATION OF HIDDEN WEB TRACKING
}

\author{
Otto Hans-Martin Lutz ${ }^{a b c}$, Jacob Leon Kröger ${ }^{a c}$, Manuel Schneiderbauer ${ }^{a d}$ and Manfred Hauswirth $^{a b c}$ \\ ${ }^{a}$ Weizenbaum Institute for the Networked Society, Berlin \\ ${ }^{\mathrm{b}}$ Fraunhofer FOKUS \\ Kaiserin-Augusta-Allee 31, 10589 Berlin, Germany \\ \{otto.lutz, manfred.hauswirth\}@fokus.fraunhofer.de \\ ${ }^{\mathrm{c}}$ Technische Universität Berlin ${ }^{\mathrm{d}}$ Humboldt-Universität Berlin
}

\begin{abstract}
Web tracking is found on $90 \%$ of common websites. It allows online behavioral analysis which can reveal insights to sensitive personal data of an individual. Most users are not aware of the amout of web tracking happening in the background. This paper contributes a sonification-based approach to raise user awareness by conveying information on web tracking through sound while the user is browsing the web.

We present a framework for live web tracking analysis, conversion to Open Sound Control events and sonification. The amount of web tracking is disclosed by sound each time data is exchanged with a web tracking host. When a connection to one of the most prevalent tracking companies is established, this is additionally indicated by a voice whispering the company name. Compared to existing approaches on web tracking sonification, we add the capability to monitor any network connection, including all browsers, applications and devices.

An initial user study with 12 participants showed empirical support for our main hypothesis: exposure to our sonification significantly raises web tracking awareness.
\end{abstract}

\section{INTRODUCTION}

Web tracking collects information about a particular user's activity on the World Wide Web. It is widely used, with some form of web tracking found on $90 \%$ of common websites, and on $60 \%$ of websites with highly privacy-critical content [1]. Although complex and extremely diverse, the ecosystem of web trackers is dominated by a small number of companies, notably by Google, Facebook and Amazon, who are inconspicuously present as third-party data collectors on many websites [2]. Recent empirical results suggest that third-party scripts owned by Google alone are present in about $80 \%$ of web traffic of the top 600 websites, and are used in a tracking context in about $40 \%$ [3].

Since a person's browsing behavior reveals insights into his or her personality, habits and sensitive aspects such as financial and

This work has been funded (in part) by the Federal Ministry of Education and Research of Germany (BMBF) under grant no. 16DII111 ("Deutsches Internet-Institut").

(c) (1) (8) This work is licensed under Creative Commons Attribution Non Commercial 4.0 International License. The full terms of the License are available at http://creativecommons.org/licenses/by-nc/4.0 medical situation or political views, web tracking may constitute a serious privacy threat [4]. Even though web tracking is seen unfavorably by the majority of internet users due to privacy concerns [5], they do not understand the full extent, the methods and possibilities of online behavioral tracking [6].

Web tracking is invisible to the user by design. Studies show that there is no sufficient awareness of web tracking [7]. We use sonification of clandestine web traffic to tracking providers as a means of raising awareness for online privacy issues. If visualization is used instead for the same objective, users must divert their visual attention from their primary task (surfing the web). Using the auditory domain, we can simultaneously communicate information in a different modality, which provides additional attention and workload resources [8]. Furthermore, sonification is suitable to present temporal data in real-time and can be shaped to convey emotional content [9, p.11, p.92].

Our contribution is a sonification-based approach to raise user awareness of web tracking which extends the possibilities of existing approaches like Soundbeam by Hutchins et al. [10]. We describe a framework for live web tracking analysis and conversion to $\mathrm{OSC}^{1}$ events, which can be used to monitor web tracking on any network connection - across all kinds of browsers, apps and devices. We discuss our system, sonification and sound design. Finally, we present results of an initial user study with 12 participants. We found empirical support for our main hypothesis: exposure to the sonification significantly raised web tracking awareness.

\section{RELATED WORK}

There is a comprehensive body of work on using sonification for network traffic monitoring to achieve higher situational awareness in a network operations center (e.g., [11, 12], systematic overview in [13]). In this context, users are network security specialists which use the auditory modality as supplementary resource to achieve their objectives in pattern, anomaly and intrusion detection. The scope of our approach, however, focuses on the average user who, in contrast to network operations professionals, is often unaware of the extent of web tracking [6]. Here, awareness refers to a general consciousness on the prevalence of web tracking. Sonification of web tracking can increase this awareness as it

${ }^{1}$ Open Sound Control, a network-based protocol for sound and media control: http://opensoundcontrol.org 
provides immediate auditory feedback to the user while he or she is browsing the internet.

Soundbeam [10] sonifies third-party connections extracted by Mozilla Lightbeam, a plug-in for the Mozilla Firefox browser. It sends data on intentionally visited websites and unintentionally visited third-parties (e.g., analytics or advertisement providers) to the SuperCollider synthesis engine via OSC. Soundbeam is designed for ensemble performance. Several users can run the software on different computers in the same network. When user B encounters a third-party element that has been identified by user A before, it is sonified for both users. This is intended to "highlight both the ubiqitousness and interconnectedness of tracking" [10].

Another related project is an earcon-based sonification of internet security threats for vision-impaired users [14]. Here, warning sounds that convey their intended meanings with little-to-no user training (e.g., casting a fishing reel to warn about a phishing attack) were used to notify users about security threats while browsing on a screen reader.

\section{FRAMEWORK DESIGN}

Our software runs in the background while the user is browsing the web. The framework comprises four stages: (1) monitoring network traffic, (2) filtering for connections to known web trackers, (3) extracting different kinds of tracking-related events, and (4) sending these events to the sound generator via OSC (see Figure 1).

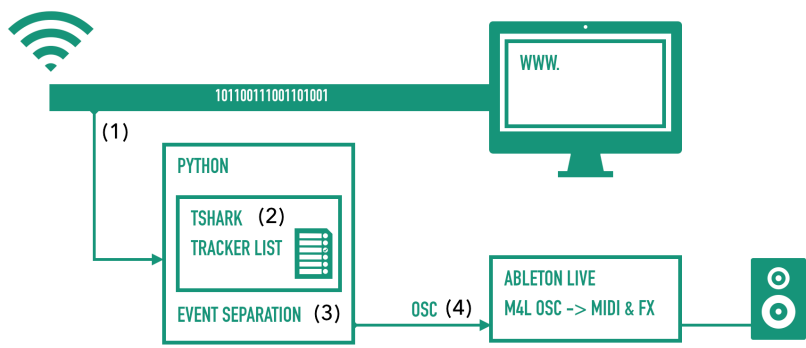

Figure 1: System overview

In the prototyping phase, we used Ableton Live [16], with a Max for Live OSC receiver for sound synthesis. We aim to switch to cross-platform (Linux supported) open source software in the future.

\subsection{Implementation}

In order to be able to intercept any network connection, we use Python to create several instances of TShark processes, a textbased version of the network protocol analyzer wireshark [15]. These processes listen to the traffic of the selected network connection. They are configured with filter lists of web tracker IP addresses, so only traffic to these addresses is analyzed in the following steps.

Tracker identification: Connections to tracker services are detected by tracker identification lists available from different sources (e.g., whotracks.me [17], easyList [18], or generated from Mozilla Lightbeam). Each list has benefits and disadvantages.
For our prototype, we used a semi-automated approach, accessing all Alexa Top 50 Websites International and Germany [19] with Mozilla Lightbeam running in the background and exporting the list of third-parties accessed. When testing the lists by browsing random websites, this semi-automatically generated list caught more third-party connections than the whotracks.me list. On the other hand, the whotracks.me list supplies a differentiation between different categories of third-parties (e.g., advertising, analytics, content delivery networks), which can provide a clearer picture of the intentions behind the third-party connection. We aim to systematically compare different tracker lists in the future.

Event separation: We configured TShark to listen to ports 80 (HTTP) and 443 (HTTPS) of the IP addresses generated from the tracker lists. We spawned separate TShark processes: a) monitoring establishment of a connection (SYN events) and b) monitoring data transferred to trackers (GET / TLS application data events). We further filter the SYN events by connections to the top 10 most prevalent trackers to further accentuate these acoustically (see Section 3.2).

All these events are stored in buffers and then sent out via OSC. As sound events which happen in close temporal proximity are not discernible anymore (precedence effect) [20], we send out the buffered events with a short pause in-between. In a heuristic pre-test, a pause of $70 \mathrm{~ms}$ turned out to provide the best balance between discering single events and an overall coherent impression.

\subsection{Sound design}

The overall purpose of our approach is raising awareness, creating interest and stimulating tought on the topic of web tracking. The auditory representation is designed to show the amount of web tracking in the background, raise interest and convey some degree of danger in order to feature the associated privacy concerns. Not only the amount of tracking is important, but the fact that a group of very few companies are present on most websites. Therefore, we aim to disclose the oligopoly of these companies as well.

When a connection to one of the top 10 tracking companies is established, we present an audio recording of the company's name in a whispered manner. Reverb is added to the whispers to intensify the spatial and suspicious impression, as a reference to the intrusion on privacy. Some of the companies are well known to users (e.g., Google, Facebook), others are less known (e.g., ComScore, criteo). The whispered names are supposed to stimulate questions about these companies as well.

Each data transfer event is presented with a short sound event. The following sound variations were designed for comparison regarding users' perception in terms of interest, curiosity, danger, and fear. We aimed to design our sounds in a way to reflect either power or fragility to convey both the power of tracking and the hidden, brittle quality it has as well. The powerful and fragile sounds were designed both in a musical and an abstract sound variation. Their numbers correspond to the sequence used in evaluation.

1. powerful and musical: low cello and tuba

2. fragile and abstract: granular synthesis

3. powerful and abstract V1: deep bleeps

4. fragile and musical: piccolo flute and violine

5. powerful and abstract V2: like V1, added delay 
A video containing both an impression of the sonic experience with our system while surfing and examples of all sound variations can be found at http://s.fhg.de/SonificationICAD2019.

\subsection{Comparison to existing approaches}

Our approach of monitoring the internet traffic itself instead of relying on the Lightbeam browser plugin extends the capabilities of Soundbeam by:

- supporting all browsers and combinations of ad / tracking blocker plug-ins.

- supporting monitoring of any physical or virtual network connection on the host computer. This enables monitoring traffic generated not only by web browsing but by apps as well.

- supporting monitoring the traffic of any device (e.g., laptop, smartphone), if we open and monitor an ad-hoc wireless network that this device connects to.

- usage and comparison of different tracker blocking lists.

- conveying the name of the tracking company by whispers.

As we have no means of identifying which addresses or links the user wants to visit, our approach does not support differentiation between intentional website visits and third-party connections. Therefore, the quality of the tracker identification list is an essential factor for a reliable result.

For now, we do not support ensemble performance as we currently aim to make an individual user aware of the tracking he or she personally is subjected to. To create a multi-user experience, the capability for sending OSC events to different computers in the network can be added to our framework.

\section{EVALUATION}

\subsection{Study design and hypothesis}

We conducted an initial user study with 12 participants (6 male, 5 female, 1 no gender stated) with an age range between 23 and 36 years, mean age was 28.9 years. In a within-subjects design, we presented the recordings of five different sound variations in a classroom setting. Each recording represented the sonification of accessing the same website. It showed the actual sonic experience while surfing, consisting of several single bleeps occurring shortly after each other. Whispering of the tracker names was muted in order to set focus on the tonal quality of the sonified events. After each sound variation, participants filled out a questionnaire regarding the perceived emotional qualities of the respective sonic experience. We asked participants to rate their overall auditory impression of the sound playback (as if visiting a website), not the single sound elements. At the end, we presented all sound variations again and asked participants to state their favorite.

For the emotional qualities of the sounds, we asked participants to rank each sound between the following poles on a fourpoint likert scale. For statstical analysis, we assigned the numbers $(-2,-1,1,2)$ to the scale items.

- innocent (-2) to dangerous (2)

- relaxing (-2) to frightening (2)

- boring (-2) to interesting (2)

- indifferent (-2) to curious (2)
As we designed the system to raise awareness, our main hypothesis is that the awareness regarding web tracking gets higher after exposure to the sonification. We assessed awareness before and after the sonification experience each with a five-point likert scale (low, rather low, medium, rather high, high).
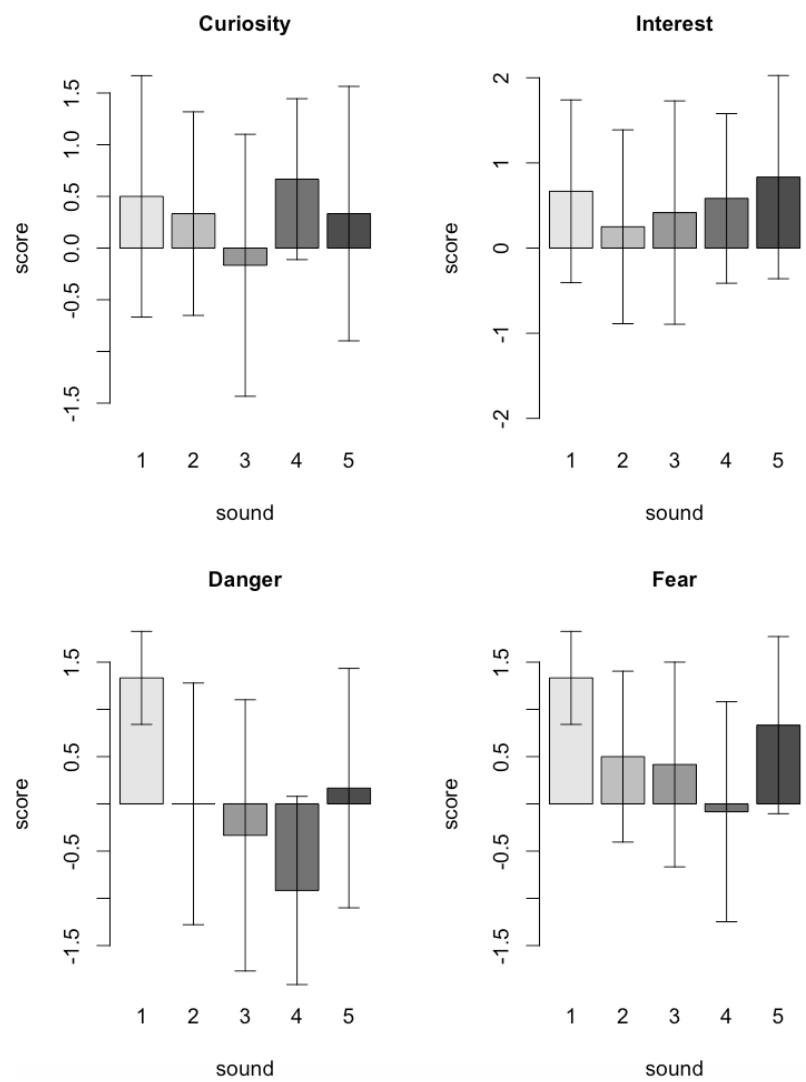

Figure 2: Emotional content of the sound variations. Error bars in plot: +/- one standard deviation

\subsection{Results}

As Shapiro-Wilk normality tests showed that normal distributions cannot be assumed in our sample, we performed a one-sided Wilcoxon signed rank test with continuity correction (see [21, p. 977]) to assess the differences between awareness scores prior to and after exposition to the sonification. The test results support our main hypothesis: Awareness levels were significantly higher after exposure to the sonification than before (mean $_{\text {before }}=$ 0.75, mean $_{\text {after }}=1.25, p=0.024, r=-0.652$ ).

Results on the emotional qualities curiosity, interest, danger and fear were less distinct and not significant (see Table 1 and Figure 2). Hence, all statements on the emotional qualities of the sounds are descriptive only. For sound 1 (low cello and tuba), danger and fear ratings were both high in mean and with a smaller standard deviation compared to the other sounds. Interestingly, sound 4 (piccolo flute and violin) was perceived least dangerous, but raised the most curiosity. Sound 1 was stated most often as favorite (five times), followed by sounds 4 and 5 (three times each). 


\begin{tabular}{lccccc}
\hline \hline Sound variation: & 1 & 2 & 3 & 4 & 5 \\
\hline mean(curiosity) & 0.500 & 0.333 & -0.167 & 0.667 & 0.333 \\
sd(curiosity) & 1.168 & 0.985 & 1.267 & 0.778 & 1.231 \\
mean(interest) & 0.667 & 0.250 & 0.417 & 0.583 & 0.833 \\
sd(interest) & 1.073 & 1.138 & 1.311 & 0.996 & 1.193 \\
mean(danger) & 1.333 & 0 & -0.333 & -0.917 & 0.167 \\
sd(danger) & 0.492 & 1.279 & 1.435 & 0.996 & 1.267 \\
mean(fear) & 1.333 & 0.500 & 0.417 & -0.083 & 0.833 \\
sd(fear) & 0.492 & 0.905 & 1.084 & 1.165 & 0.937 \\
\hline
\end{tabular}

Table 1: Sound variations: Means and standard deviations of emotional content scores

\section{DISCUSSION}

The initial user study has limitations: Most notably, as the sounds were presented in a classroom setting, a sequence effect is expected. Future studies will benefit from individual presentation via headphones and randomisation of the sound variations. Adjectives of the emotional quality poles were not selected from standardized test batteries on emotional content. Additionally, the sample size of 12 participants was quite small. Nevertheless, some effect of the sonification experience on web tracking awareness could be shown.

\section{FUTURE RESEARCH}

As our initial results are encouraging, we will continue and extend our work in the following ways: First, we aim to set it up in a way that supports connecting a user's own device (laptop, smartphone) to a special wireless network we provide and monitor. By this, we allow users to explore the tracking sounds of their own browser or app configuration. We are also looking into porting the framework to a small computer like the Raspberry Pi [22]. This can ease the usage of our system in installations in public. Then, we plan to conduct a larger user study that assesses the impact of our approach to web tracking awareness in the field.

Future research questions regarding sound design are manifold: We aim to disclose not only the amount of web tracking, but the oligopoly of the tracking companies as well. So far, we approached this with the tracker name whispering when connecting initially. In future, we want to design signature sounds for each company, so the corresponding single events can be linked to these companies. Another significant step is moving on from producing the sounds in Ableton Live to a model-based sonification. Additionally, incorporating the spatial domain can help conveying tracker parameters by placement in the virtual room.

\section{ACKNOWLEDGMENT}

The authors want to thank Jan Maria Kopankiewicz for his support with implementation.

\section{REFERENCES}

[1] S. Schelter and J. Kunegis, "On the Ubiquity of Web Tracking: Insights from a Billion-Page Web Crawl," pp. 53-66, 2016. [Online]. Available: http://arxiv.org/abs/1607. 07403
[2] S. Macbeth, "Tracking the Trackers: Analysing the global tracking landscape with GhostRank," Cliqz GmbH, Tech. Rep., 2017.

[3] A. Karaj, S. Macbeth, R. Berson, and J. M. Pujol, "WhoTracks.Me: Monitoring the online tracking landscape at scale," pp. 1-15, 2018. [Online]. Available: http: //arxiv.org/abs/1804.08959

[4] A. Acquisti, L. Brandimarte, and G. Loewenstein, "Privacy and human behavior in the age of information," Science, vol. 347, no. 6221, pp. 509-514, 2015.

[5] K. Purcell, J. Brenner, and L. Rainie, "Search engine use 2012," Search, 2012.

[6] T. Bujlow, V. Carela-Espanol, B. R. Lee, and P. Barlet-Ros, "A Survey on Web Tracking: Mechanisms, Implications, and Defenses," Proceedings of the IEEE, vol. 105, no. 8, pp. 1476-1510, 2017.

[7] W. Thode, J. Griesbaum, and T. Mandl, “'”I would have never allowed it": User Perception of Third-party Tracking and Implications for Display Advertising," in Proc. International Symposium on Information Science, 2015.

[8] C. D. Wickens, "Multiple resources and mental workload," Human factors, vol. 50, no. 3, pp. 449-55, 2008.

[9] T. Hermann, A. Hunt, and J. G. Neuhoff, The Sonification Handbook, 1st ed. Berlin: Logos Publishing House, 2011.

[10] C. Hutchins, H. Ballweg, S. Knotts, J. Hummel, and A. Roberts, "Soundbeam: A Platform for Sonyfing Web Tracking," Proceedings of the International Conference on New Interfaces for Musical Expression, pp. 497-498, 2014.

[11] M. Ballora, N. Giacobe, and D. Hall, "Songs of cyberspace: an update on sonifications of network traffic to support situational awareness," Proc. SPIE Defense + Commercial Sensing, vol. 8064, pp. 1-6, 2011.

[12] M. Debashi and P. Vickers, "Sonification of network traffic flow for monitoring and situational awareness," PLOS ONE, vol. 13, no. 4, pp. 1-31, 2018.

[13] L. Axon, S. Creese, M. Goldsmith, and J. R. C. Nurse, "Reflecting on the Use of Sonification for Network Monitoring," Proc. SECURWARE 2016, pp. 254-261, 2016.

[14] A. Siami Namin, R. Hewett, K. S. Jones, and R. Pogrund, "Sonifying Internet Security Threats," Proc. 2016 Conference on Human Factors in Computing Systems Extended Abstracts, pp. 2306-2313, 2016.

[15] https://www.wireshark.org, [Accessed 20.04.2019].

[16] https://www.ableton.com, [Accessed 20.04.2019].

[17] https://github.com/cliqz-oss/whotracks.me, [Accessed 20.04.2019].

[18] https://github.com/easylist, [Accessed 20.04.2019].

[19] https://www.alexa.com/topsites, [Accessed 20.04.2019].

[20] H. Wallach, E. B. Newman, and M. R. Rosenzweig, "A Precedence Effect in Sound Localization," The Journal of the Acoustical Society of America, vol. 21, p. 468, 1949.

[21] A. Field, J. Miles, and Z. Field, Discovering Statistics Using $R$. SAGE Publications, 2012.

[22] https://raspberrypi.org, [Accessed 20.04.2019]. 